\title{
Methods to improve rehabilitation of patients following breast cancer surgery: a review of systematic reviews
}

This article was published in the following Dove Press journal:

Breast Cancer: Targets and Therapy

II March 2015

Number of times this article has been viewed

\section{Siew Yim Loh \\ Aisya Nadia Musa}

Department of Rehabilitation Medicine, Faculty of Medicine, University of Malaya, Kuala Lumpur, Malaysia
Correspondence: Siew Yim Loh Department of Rehabilitation Medicine, Faculty of Medicine, University of Malaya, Kuala Lumpur, Malaysia.

Email syloh@um.edu.my; siewyimloh@ yahoo.com
Context: Breast cancer is the most prevalent cancer amongst women but it has the highest survival rates amongst all cancer. Rehabilitation therapy of post-treatment effects from cancer and its treatment is needed to improve functioning and quality of life. This review investigated the range of methods for improving physical, psychosocial, occupational, and social wellbeing in women with breast cancer after receiving breast cancer surgery.

Method: A search for articles published in English between the years 2009 and 2014 was carried out using The Cochrane Database of Systematic Reviews, the Database of Abstracts of Reviews of Effects, PubMed, and ScienceDirect. Search terms included: 'breast cancer', 'breast carcinoma', 'surgery', 'mastectomy', 'lumpectomy', ‘breast conservation', 'axillary lymph node dissection', 'rehabilitation', 'therapy', 'physiotherapy', 'occupational therapy', 'psychological', 'psychosocial', 'psychotherapy', 'exercise', 'physical activity', 'cognitive', 'occupational', 'alternative', 'complementary', and 'systematic review'.

Study selection: Systematic reviews on the effectiveness of rehabilitation methods in improving post-operative physical, and psychological outcomes for breast cancer were selected. Sixteen articles met all the eligibility criteria and were included in the review.

Data extraction: Included review year, study aim, total number of participants included, and results.

Data synthesis: Evidence for exercise rehabilitation is predominantly in the improvement of shoulder mobility and limb strength. Inconclusive results exist for a range of rehabilitation methods (physical, psycho-education, nutritional, alternative-complementary methods) for addressing the domains of psychosocial, cognitive, and occupational outcomes.

Conclusion: There is good evidence for narrowly-focused exercise rehabilitation in improving physical outcome particularly for shoulder mobility and lymphedema. There were inconclusive results for methods to improve psychosocial, cognitive, and occupational outcomes. There were no reviews on broader performance areas and lifestyle factors to enable effective living after treatment. The review suggests that comprehensiveness and effectiveness of post-operative breast cancer rehabilitation should consider patients' self-management approaches towards lifestyle redesign, and incorporate health promotion aspects, in light of the fact that breast cancer is now taking the form of a chronic illness with longer survivorship years.

Keywords: breast cancer surgery, rehabilitation methods, symptom-management, quality of life, lifestyle redesign, self-management

\section{Introduction}

Breast cancer incidences ranges from 19.3 per 100,000 women in Eastern Africa to 89.7 per 100,000 women in Western Europe and about 40 per 100,000 in 
developing countries. ${ }^{1}$ The 5 -year relative survival rates for breast cancer in the US have improved dramatically from $63 \%$ in the 1960 s to $90 \%$ in $2011 .^{2}$ In Malaysia, the survival rates estimated in 2009 was 43.5\%, with Malay, Chinese, and Indians, and Malays having 5-year survival rates of $39.7 \%, 48.2 \%$, and $47.2 \%$ respectively, and the rates have also improved annually. The number of breast cancer survivors has increased dramatically as a result of early detection, better treatment, and various multidisciplinary rehabilitation methods. ${ }^{3,4}$ However, improved survival rate of breast cancers also comes with numerous side effects from the cancer and its treatment. There is a need for comprehensive rehabilitation methods to address the many impacts of the long-term effects of this treatment, including the less recognized cognitive impairments ${ }^{5}$ to improve survivors' global functioning.

Surgery is usually conducted with the goal to completely remove breast tumors, either by mastectomy or lumpectomy, and to assess the status of the axillary lymph node, either through sentinel lymph node biopsy (SLNB) or axillary lymph node dissection (ALND). ${ }^{6,7}$ Often, post-surgery rehabilitation focuses on the more obvious side effects, with pain and physical impairments being reported as the most debilitating complications after surgery. Therefore, commonly reported are upper body symptoms such as shoulder functions, breast/arm swelling (or lymphedema) with deformity, impairment of functionality, physical discomfort and numbness of the skin on upper arm and impaired arm. ${ }^{8-10}$ Reports from lymphedema studies showed it occurs in $10 \%-50 \%$ of women who underwent ALND and among $5 \%-20 \%$ of women who underwent SLNB. ${ }^{11}$ Post-operative, long-term pain has also been reported in $12 \%-50 \%$ of women with breast cancer, usually due to nerve injuries during surgery. ${ }^{12}$

Prevalence of cognitive impairment occurs in 10\%-50\% of women. ${ }^{7,13}$ It impacts on daily living performances (activities of daily living, work, and leisure tasks) and the overall quality of life (QoL), but is often ignored, partly because its cause cannot be identified. Furthermore, occupational outcomes, such as time needed to return to work, work absenteeism, and sick leave or employment status is also a concern of breast cancer survivors. ${ }^{14-16}$ Emotional distress caused by shifts in social support, and fear of recurrence and death has also impacted women's wellbeing. ${ }^{17,18}$ However, the rehabilitation is less commonly reported and includes the less obvious psychosocial functioning, including anxiety and depression, and where esthetic deformity or affected body image have been implicated leading to poor coping strategies. ${ }^{19}$ As such, these after-effects from the post-operative procedures and adjuvant therapies can lead to a compromised QoL. ${ }^{20}$ Holistic rehabilitation including health-promotion and health-prevention strategies, and via early Occupational therapy involvements is warranted for effective living with breast cancer.

\section{Description of intervention}

Management of long-term side effects of breast cancer treatment is important to improve QoL of breast cancer survivors. ${ }^{21}$ Optimal rehabilitation includes the inputs from the various health professionals to help remediate and restore the impaired physical, psycho-social, and occupational functioning of women with breast cancer. ${ }^{22}$ Some of these methods include physical-therapy, ${ }^{23,24}$ exercise interventions, ${ }^{25,26}$ psychological therapies such as psycho-education, ${ }^{27,28}$ occupational therapy, ${ }^{29,30}$ nutritional rehabilitation, ${ }^{31}$ alternative rehabilitation such as yoga, music therapy, ${ }^{32,33}$ and complex rehabilitation. ${ }^{34}$

\section{Aim}

This review aims to examine systematic reviews on the rehabilitation methods for post-operative women with breast cancer, with a view on the comprehensiveness of these methods used, and if they consider breast cancer as a chronic illness. The findings may help inform suitable treatment decisions towards post-operative complications and the after-effects so that survivors can live for indefinite periods, with breast cancer taking a form of chronic illness.

\section{Methods \\ Search terms}

Systematic reviews were searched in four databases, restricted to full-text English language publications, which were published between January 2009 and October 2014, on adult women with breast cancer including The Cochrane Database of Systematic Reviews, the Database of Abstracts of Reviews of Effects, PubMed, and ScienceDirect.

The titles, abstracts, and keywords were searched for the following terms in order to identify the required articles: 'breast cancer', 'breast carcinoma', 'surgery', 'rehabilitation', 'treatment', 'therapy', 'physical therapy', 'occupational therapy', 'psychological', 'psychosocial', 'exercise', 'physical activity', 'cognitive', 'occupational', 'alternative', 'complementary', and 'systematic review'. Search terms were identified by means of the inclusion and exclusion criteria specified in the PICOS (Population, Intervention, Comparator, Outcomes, and Study designs) table (Table 1). Boolean operators "AND", and "OR" and search 
Table I PICOS inclusion and exclusion criteria

\begin{tabular}{|c|c|c|}
\hline & Inclusion criteria & Exclusion criteria \\
\hline \multirow[t]{5}{*}{ Population } & Adults aged 18 years and over & Children and adolescents \\
\hline & Women with post-operation breast cancer & Participants involving men \\
\hline & & Co-morbidity physical (such as severe cardiac \\
\hline & & disease and hypertension) and psychiatric illnesses \\
\hline & & Women with metastatic cancer \\
\hline \multirow[t]{9}{*}{ Interventions } & Physical and occupational therapy (eg, complex decongestive therapy, manual & Pharmacological therapies \\
\hline & lymph drainage, standard physiotherapy, occupational therapy) & Alternative medicine - eg, Chinese medicine \\
\hline & Exercise (eg, exercise therapy, home based exercise routine, weight training) & herbal \\
\hline & Psychosocial (eg, CBT, psycho-education) & \\
\hline & Alternative/complementary (eg, hypnotherapy, acupuncture, homeopathy, yoga) & \\
\hline & Nutritional (eg, dietary regime) & \\
\hline & Complex (eg, combination of psycho-education and nutrition etc rehabilitation) & \\
\hline & Reviews on pharmacological therapies and alternative medicine may be & \\
\hline & included if other rehabilitation methods mentioned above were also reviewed & \\
\hline Comparator & Systematic reviews of RCT interventions, cross-sectional studies, qualitative & None \\
\hline (eg, control) & studies with or without control/comparison groups & \\
\hline \multirow[t]{4}{*}{ Outcomes } & Physical (eg, lymphedema, shoulder mobility) & None \\
\hline & Psychosocial (eg, anxiety, depression, affect/mood, QoL) & \\
\hline & Occupational (eg, return to work, occupational gap, lifestyle) & \\
\hline & Cognition (eg, “chemo-brain”, cognitive functioning) & \\
\hline \multirow[t]{7}{*}{ Study design } & $\begin{array}{l}\text { Systematic reviews (systematic reviews of RCTs, non-randomized studies, } \\
\text { cross-sectional, qualitative studies, etc) }\end{array}$ & $\begin{array}{l}\text { Individual studies (RCTs, non-randomized studies, } \\
\text { cross-sectional, qualitative, etc) }\end{array}$ \\
\hline & English language & Meta-analysis of studies \\
\hline & Dated from January 2009 to October 2014 & Systematic reviews dated before January 2009 \\
\hline & & Systematic reviews with too few included studies \\
\hline & & (less than four included studies) \\
\hline & & Systematic reviews with too few databases \\
\hline & & searched (less than three electronic searches) \\
\hline
\end{tabular}

Abbreviations: RCTs, randomized controlled trials; PICOS, Population, Intervention, Comparator, Outcomes, and Study designs; CBT, cognitive behavior therapy; QoL, quality of life.

filter "asterisks (*)" were used together with the search terms to ensure all keyword variations were searched. Grey literature was excluded.

\section{Selection of reviews}

The electronic searches resulted in a number of studies uncovered from each database and was recorded immediately as shown in Figure 1. The online reference manager (EndNote) was used to remove any duplicates. Reviews were reviewed by one reviewer (ANM) to first assess their eligibility by reading the title and the abstract of each study.

\section{Participants}

Systematic reviews which include a sample of post-surgery breast cancer adults (aged 18 years and over) were included. Reviews were excluded if the sample had other types of non-breast cancer.

\section{Intervention}

Systematic reviews on the effectiveness of single/combined (complex) rehabilitation eg, physical/occupational therapy, exercise, psychological, occupational, cognitive, nutrition, and alternative methods for post-operative breast cancer. The interventions included:

- Physical therapy - complex decongestive therapy, manual lymph drainage (MLD), standard physiotherapy, occupational therapy etc.

- Physical exercise - home based and instructed exercise.

- Psychosocial - cognitive behavior therapy (CBT), psycho-education, etc.

- Nutritional - change in dietary habits, dietary regime, etc.

- Alternative - yoga, music therapy, acupuncture, etc.

- Complex - combined interventions eg, counseling and exercise.

\section{Types of studies}

All published systematic reviews in the English language, on rehabilitation programs (for a combination of dysfunctions) were selected. The programs could have been physical or exercise or psychological or cognitive or occupational or nutritional or alternative or complex rehabilitation. There were no restrictions on the type of systematic 


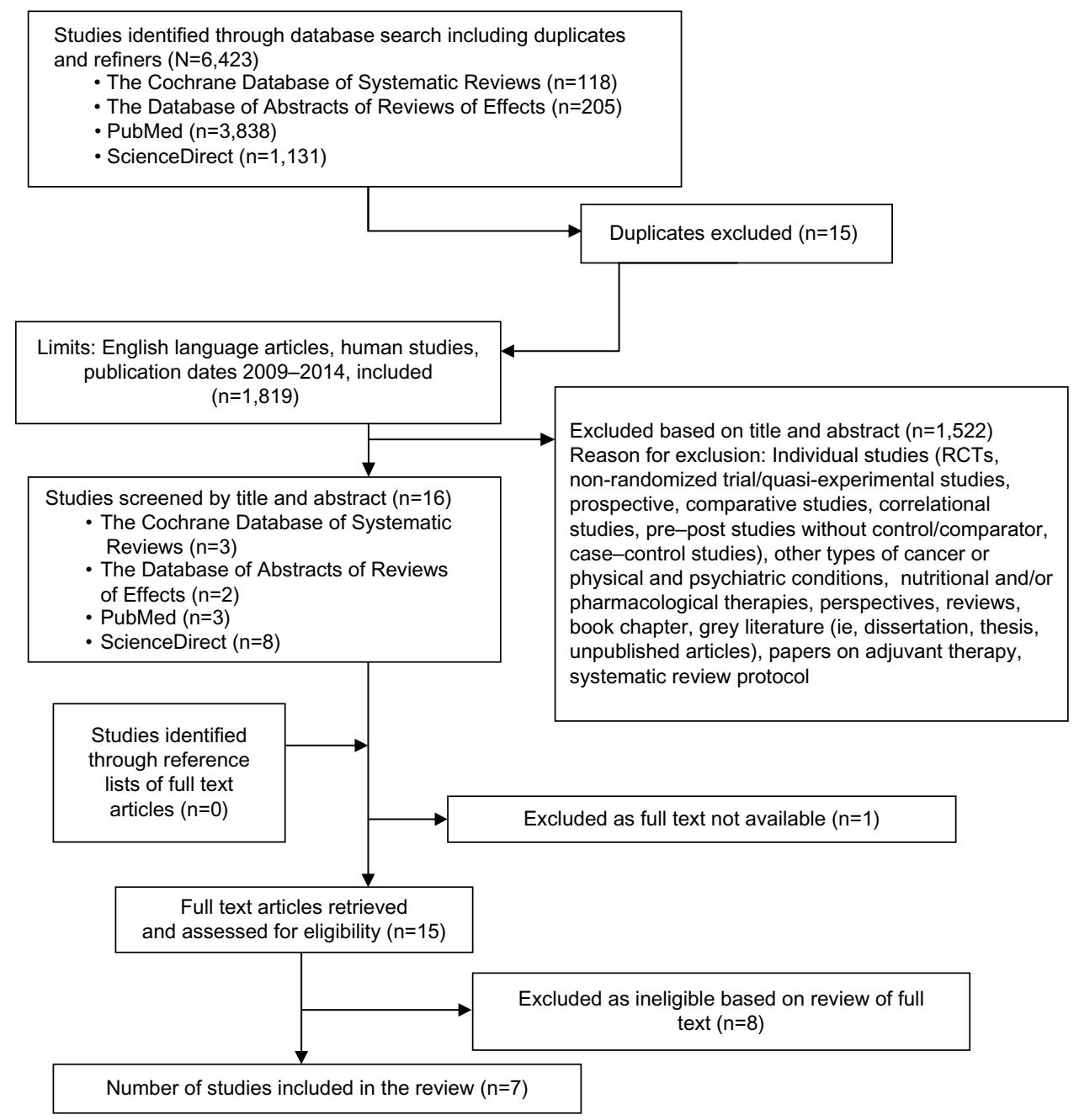

Figure I Flow diagram of systematic review process. Abbreviation: RCTs, randomized controlled trials.

review, ie, systematic reviews of randomized controlled trials (RCTs), uncontrolled trials, non-randomized studies, qualitative, etc, provided that the aim of the study was to investigate the effects of either single or combinational rehabilitation methods/programs for post-operative patients with breast cancer. Systematic reviews were excluded if they focused on one narrow aspect or just one modality (eg, just specifically arm lymphedema as the outcome) and/or if there is less than four studies in the review paper, or it utilized less than three databases to search for individual studies.

\section{Outcome measures}

Systematic reviews on the physical, cognitive, occupational, and psychological outcomes in post-operative breast cancer patients were included:

- Physical outcomes - shoulder mobility, lymphedema, wound healing, fatigue, etc.
- Psychological outcomes - QoL, anxiety, depression, mood, and stress.

- Cognitive outcomes - memory, attentiveness, "chemobrain", etc.

- Occupational outcomes - return to work, absenteeism, etc.

- Lifestyle redesign - preventive and health promotion methods.

\section{Eligibility criteria}

The present systematic review included published systematic review articles in the English language between the years 2009 and 2014. Unpublished reviews (grey literatures) were not included in the review. Inclusion criteria were kept relatively broad to ensure comprehensiveness in assessing the various rehabilitation methods reviewed in previous systematic reviews. 


\section{Data extraction and synthesis of results}

Reviews were selected based on inclusion and exclusion criteria as depicted in the flow diagram (Figure 1). All relevant reviews accessed were followed-up to establish inclusion. The information extracted included data from the data extraction table (Table $\mathrm{S} 1$ ) guided by a previous systematic review of reviews. ${ }^{35}$ The data were extracted by one researcher (ANM) which was guided, assessed, and reviewed by a senior researcher (SYL). It was expected that there would be heterogeneity in the outcomes measured.

\section{Risk of bias in individual studies}

Methodological quality of included systematic reviews was independently rated according to the "assessment of multiple systematic reviews" (AMSTAR) tool. ${ }^{36}$ Responses of the AMSTAR tool are 'yes', 'no', 'can't answer', or 'not applicable', with yes being rated as ' 1 ', and 'no', 'can't answer', or 'not applicable' rated as ' 0 '. Based on this scale, reviews were rated as 'low', 'moderate' or 'high' quality. The domains identified in the 11-item tool are presented in Table 2.

\section{Results}

\section{Study selection}

Figure 1 is the flow chart of the systematic review process from four databases (Cochrane Database of Systematic Reviews, Database of Abstracts of Reviews of Effects, PubMed, and ScienceDirect), which yielded seven full-text systematic reviews, ${ }^{7,22,37-41}$ and excluded eight full-text reviews. ${ }^{8,42-48}$ Reasons for the exclusion of reviews are stated in the screening inclusion and exclusion table (Table S1).

Table 2 A I I-item "assessment of multiple systematic reviews" (AMSTAR) for assessing systematic reviews

\section{The AMSTAR tool}

I. Was an "a priori" design provided?

2. Was there duplicate study selection and data extraction?

3. Was a comprehensive literature search performed? At least two electronic sources, include years and databases used (eg, Central, EMBASE, and MEDLINE).

4. Was the status of publication (ie, grey literature) used as an inclusion criterion?

5. Was a list of studies (included and excluded) provided?

6. Were the characteristics of the included studies provided?

7. Was the scientific quality of the included studies assessed and documented?

8. Was the scientific quality of the included studies used appropriately in formulating conclusions?

9. Were the methods used to combine the findings of studies appropriate?

10. Was the likelihood of publication bias assessed?

II. Was the conflict of interest included?
Table 3 shows the AMSTAR tool for assessing methodological quality or rigor of each review included. Based on the AMSTAR tool, three out of the seven reviews reported were of good methodological quality, ${ }^{22,37,38}$ with three RCTs of medium quality, ${ }^{7,39,40}$ and one review of low quality. ${ }^{41}$ All but one systematic review ${ }^{41}$ had ensured at least two independent researchers had been involved in data extraction, or at least one researcher had checked the other's work. All, but one review ${ }^{7}$ had ensured a comprehensive literature search (ie, a search strategy using more than two databases and one supplementary strategy that uses review of references in individual studies). Only one systematic review ${ }^{38}$ had searched for and attained unpublished or grey literature. Three reviews ${ }^{22,37,38}$ had ensured a list of included and excluded studies, with reasons for inclusion and exclusion provided. Methodological quality assessments of included studies were measured in all, but one review. ${ }^{41}$ However, only two reviews ${ }^{38,40}$ had considered the methodological quality of each individual study in carrying out a conclusion or recommendation of rehabilitation methods. All, but two reviews ${ }^{7,41}$ had assessed heterogeneity of included studies reviewed. Publication bias was assessed. In two reviews ${ }^{38,39}$ publication bias could not be assessed since the number of included studies was less than 20 .

\section{Characteristics of included reviews}

A summary of the review is presented in Table 4, outlining its year of publication, aim, search strategy used, inclusion and exclusion criteria, number and design of included studies in each review, total number and age of participants, and outcomes measured.

\section{Description of methods}

The selected systematic reviews vary considerably in terms of the designs of the studies included, type of rehabilitation methods, and search strategy (databases used, publication years and language restriction, and search terms) (Table S2). Five out of the seven systematic reviews were RCTs. ${ }^{22,37-40}$ One review included both controlled and uncontrolled quantitative trials. ${ }^{41}$ Another study included qualitative studies only. ${ }^{7}$ Three reviews had specifically investigated physical rehabilitation methods: two papers were on the efficacy of various types of exercise, ${ }^{37,38}$ whilst one was on weight training exercises. ${ }^{39}$ One review investigated psychosocial interventions ${ }^{40}$ another the efficacy of occupational rehabilitation on women with breast cancer, ${ }^{41}$ and one explored cognitive functioning. ${ }^{7}$ Only the review by Juvet et $\mathrm{al}^{22}$ covered a holistic range of rehabilitation 
Table 3 AMSTAR (assessment of multiple systematic reviews) checklist

\begin{tabular}{llllllll}
\hline The AMSTAR & ${\text { Chan et } \mathrm{al}^{37}}^{7}$ & ${\text { McNeely et } \mathrm{al}^{38}}^{38}$ & Paramanandam and & Fors et al $^{40}$ & Hoving et al $^{41}$ & Juvet et al $^{22}$ & Selamat et al $^{7}$ \\
Tool & $(2010)$ & $(2010)$ & Roberts $^{39}(2014)$ & $(2011)$ & $(2009)$ & $(2009)$ & $(2014)$
\end{tabular}

Tool

(2010)

Roberts $^{39}$ (2014)

(20II)

(2009)

(2009)

(2014)

I. Was an "a priori" design provided?

Yes $\sqrt{ } \quad \sqrt{ }$

No

$\sqrt{ } \sqrt{ }$

Can't answer -

Not applicable -

2. Was there duplicate study selection and data extraction?

Yes

No

Can't answer -

Not applicable -

3. Was a comprehensive literature search performed?

Yes V V

No -

Can't answer -

Not applicable -

4. Was the status of publication (ie, grey literature) used as an inclusion criterion?

Yes - $\quad \sqrt{ }$

No $\quad \sqrt{ }$

Can't answer -

Not applicable -

5. Was a list of studies (included and excluded) provided?

Yes $\sqrt{ }$

No

Can't answer -

Not applicable -

6. Were the characteristics of the included studies provided?

Yes $\sqrt{ }$

No

Can't answer -

Not applicable -

7. Was the scientific quality of the included studies assessed and documented?

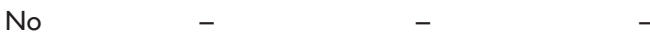

Can't answer -

Not applicable -

8. Was the scientific quality of the included studies used appropriately in formulating conclusions?

$\begin{array}{llll}\text { Yes } & - & \sqrt{ } & - \\ \text { No } & \sqrt{ } & - & \sqrt{ } \\ \text { Can't answer } & - & - & - \\ \text { Not applicable } & - & - & - \\ \text { Were the methods used to combine the findings of studies }\end{array}$

9. Were the methods used to combine the findings of studies appropriate?

Yes $\sqrt{ } \quad \sqrt{ }$

No

Can't answer

Not applicable

10. Was the likelihood of publication bias assessed?

Yes

No

Can't answer

Not applicable -

II. Was the conflict of interest included?

Yes $V$

No

Can't answer -

Not applicable -

Overall score

8/II II/II

High quality High quality $\checkmark$

$-$

$-$

$\sqrt{ }$

$-$

$-$

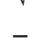

$-$

$-$

$\sqrt{ }$

$-$

.

/II

Moderate quality

\section{$\sqrt{ } \quad \sqrt{ }$}
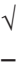

$-$

$-$ $\sqrt{ }$ $\sqrt{ }$ - 


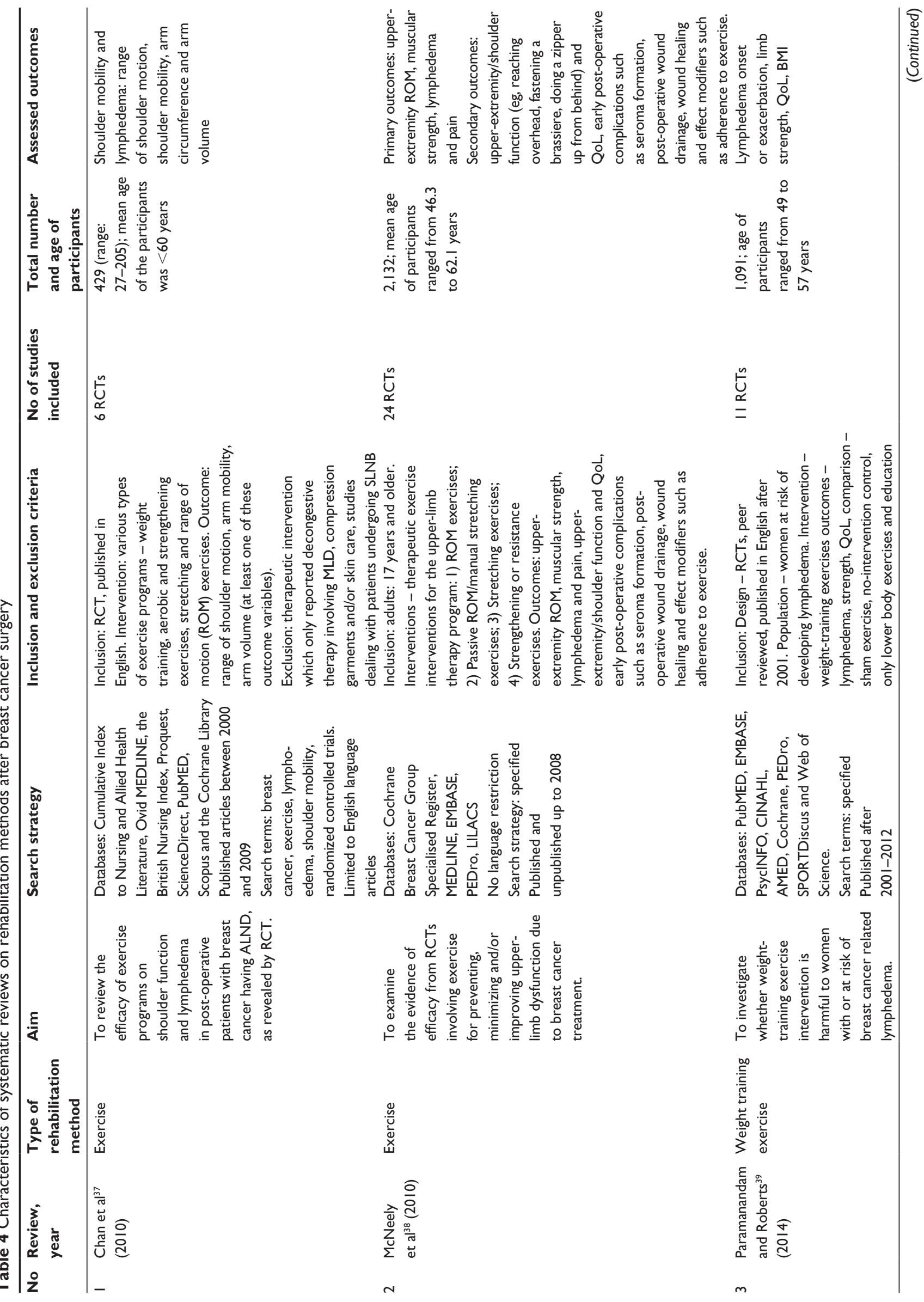




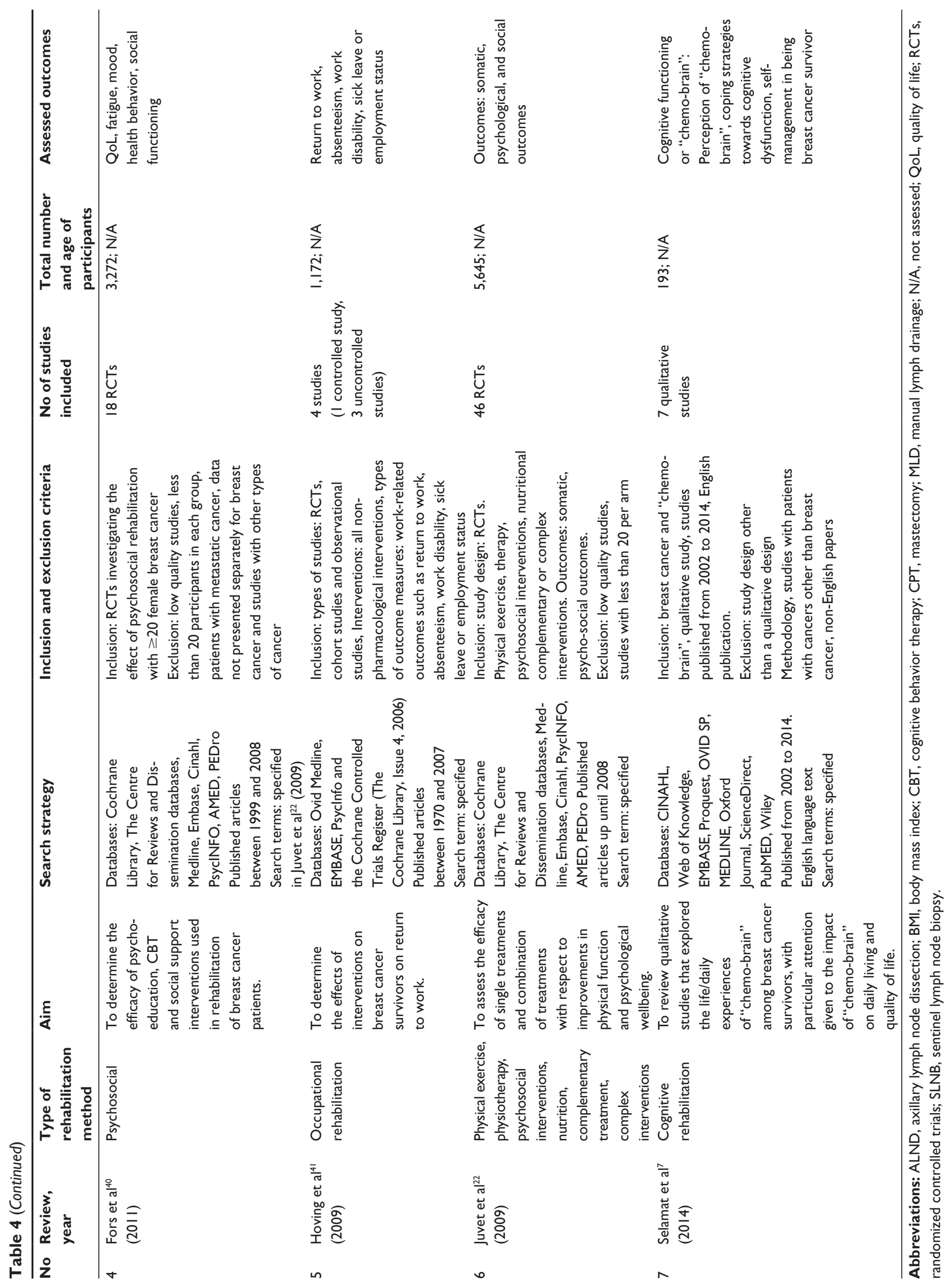


methods - physical activity rehabilitation, psychosocial rehabilitation, nutritional rehabilitation, complementary or alternative rehabilitation (ie, yoga, music therapy, etc), and complex (psycho-education plus counseling) rehabilitation for women after breast cancer. ${ }^{22}$

For each of the resulted review, we had searched in more than one database for individual studies (refer to Table 4). Restriction of publication years and language varied across the seven reviews. Chan et $\mathrm{al}^{37}$ included published articles between the years 2000 and 2009. Paramanandam and Roberts ${ }^{39}$ restricted review to articles dated between 2001 and 2012, Fors et a ${ }^{40}$ included published articles between the years 1999 and 2008, Hoving et al ${ }^{41}$ included articles between the years of 1970 and 2007, Juvet et a ${ }^{22}$ included published articles up until 2008, and Selamat et $\mathrm{al}^{7}$ included articles restricted to those published from 2002 to 2014. One review ${ }^{38}$ included both published and unpublished literature until 2008. Three reviews ${ }^{7,37,39}$ restricted articles to the English language only. One review ${ }^{38}$ did not have any language restrictions. Three reviews ${ }^{22,40,41}$ did not specify language restrictions. All reviews specified the various search terms used.

\section{Description of participants}

The total number of participants was specified in all seven reviews. The age of participants was specified in three reviews $^{37-39}$ only. Details of the total number and age of participants are provided in Table 4 . All but three reviews ${ }^{38,39,41}$ specified exclusion criteria for participants. Chan et $\mathrm{al}^{37}$ excluded reviews which included male participants.

\section{Description of outcomes}

Physical, psychosocial, occupational, and cognitive outcomes varied. Amongst the physical outcomes assessed were: upper body symptoms (ie, shoulder function, arm movement, limb strength), ${ }^{37,38}$ risk or incidence of secondary lymphedema, ${ }^{37-39}$ fatigue, ${ }^{40}$ pain, ${ }^{38}$ seroma formation, ${ }^{38}$ wound drainage, ${ }^{38}$ physical fitness (ie, body mass index [BMI], body composition), ${ }^{22,39}$ and adherence to exercise. ${ }^{38}$ Amongst the psychosocial outcomes were QoL, ${ }^{38-40}$ mood, health behavior, and social function. ${ }^{40}$ Occupational outcomes included return to work, absenteeism, work disability, sick leave or employment status measured by only one systematic review. ${ }^{41}$ Cognitive outcomes include perception of "chemo-brain", coping strategies towards cognitive dysfunction, and self-management as breast cancer survivor. Only one review ${ }^{22}$ had attempted to look at somatic, psychological, and social outcomes as a comprehensive whole.

\section{Findings Effects on physical outcomes}

Table 5 shows the results of the effects of various rehabilitation methods on physical outcomes. Five reviews had investigated the effects of rehabilitation for physical outcomes, with adequate methodological quality: three reviews were rated as having high methodological quality, ${ }^{22,37,38}$ and two were rated as having medium quality. ${ }^{39,40}$

Exercise rehabilitation showed significant improvement in shoulder movement - irrespective of type or time period of implementation ${ }^{37}$ but, early exercise was found to be more effective than delayed exercise. ${ }^{38}$ Paramanandam and Roberts ${ }^{39}$ found that gradual intensity weight training, with slow progression, improved upper and lower limb strength. More importantly, exercise did not increase risk or change in the incidence rate of lymphedema ${ }^{22,37-39}$ and complex decongestive therapy decreased the incidence of lymphedema as compared to standard physiotherapy. ${ }^{22}$ The benefits of exercise were well reported. Early exercise (versus delayed) also helps wound healing by increasing the wound drainage volume. ${ }^{38}$ There were inconclusive results for exercise interventions on BMI. 22,39 With fatigue management, psycho-education had a significant short-term benefit, ${ }^{40}$ but not cognitive behavioral therapy. ${ }^{22,40}$ With hot flashes, complementary rehabilitation (acupuncture, yoga, art therapy, or relaxation training) did not show conclusive evidence. ${ }^{22}$ Overall, the physical rehabilitation seems to focus on shoulder range of motion, fatigue, body weight, wound, and hot flashes amongst the wide range of after-effects from breast cancer and its treatment.

\section{Effects on non-physical related outcomes}

Table 6 shows the effects of rehabilitation methods on less obvious psychological, occupational, and cognitive outcomes. Of the five reviews, the methodological quality of the three reviews was rated as high quality, ${ }^{22,37,38}$ and two reviews ${ }^{39,40}$ were rated as medium quality.

QoL was assessed in three reviews ${ }^{22,39,40}$ as an outcome of exercise rehabilitation, ${ }^{22,39} \mathrm{CBT}^{40}$ and psycho-education ${ }^{22,40}$ albeit with inconclusive results. One review ${ }^{22}$ found inconclusive results on the benefit of both complementary and complex decongestive therapy on QoL. Health behaviors and social function and coping were assessed in two reviews ${ }^{22,40}$ with inconclusive results. There were inconclusive results of social and emotional support interventions in two reviews. ${ }^{22,40}$ Mood outcomes such as anxiety, event related distress, and depression assessed in two reviews ${ }^{22,40}$ found that psychoeducation, CBT, and social and emotional support interventions yield inconclusive results towards improving mood. There was some evidence that complementary intervention 


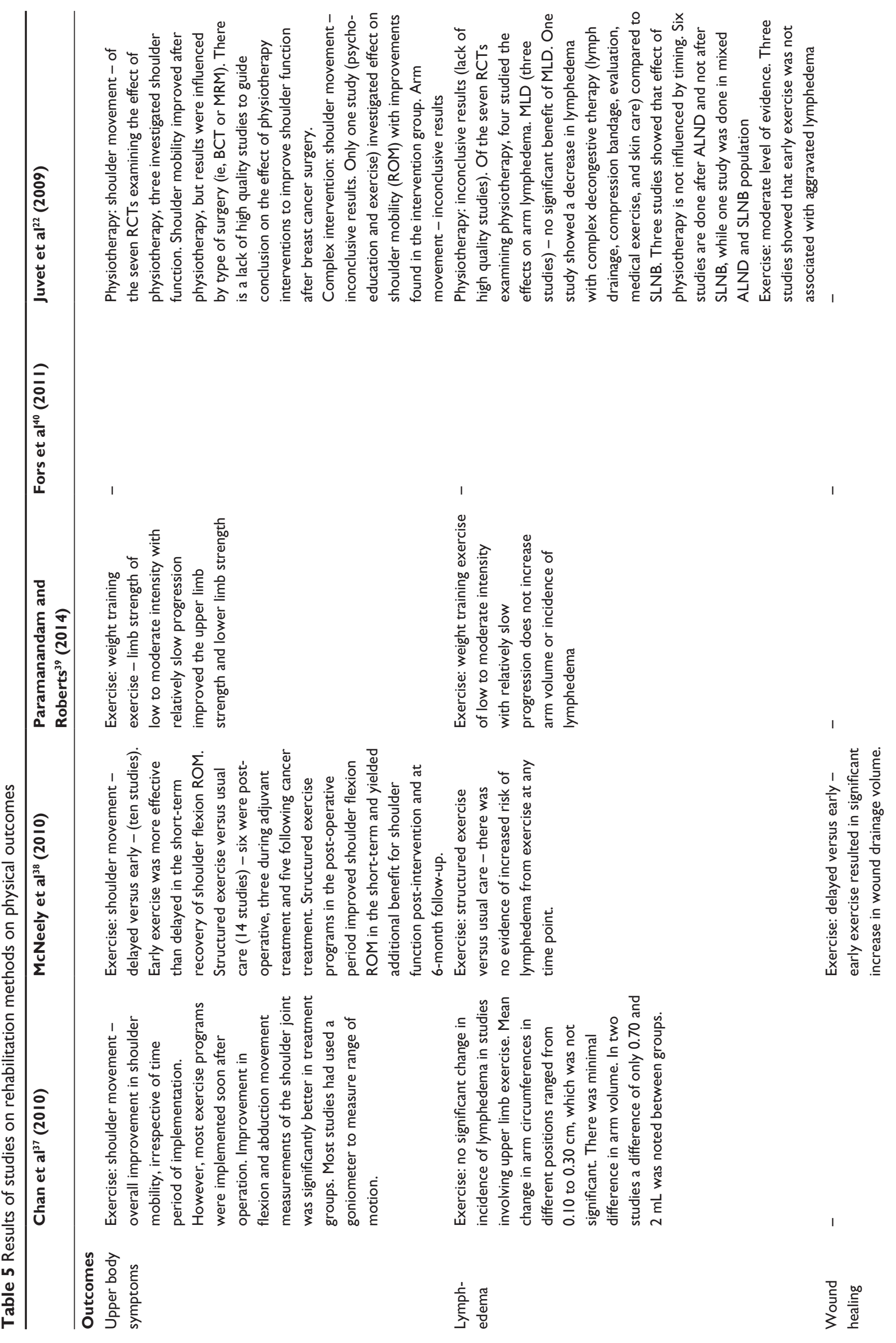




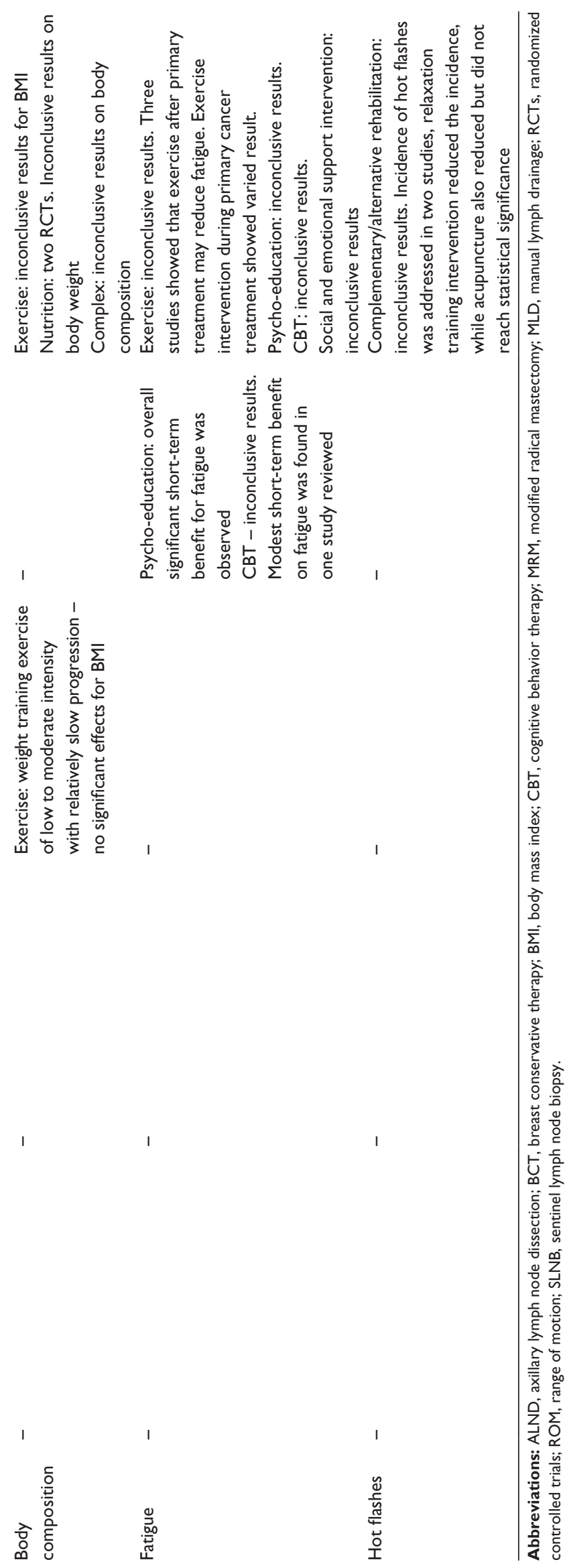

after primary breast cancer treatment was found to have a small effect on mood outcomes. ${ }^{22}$

\section{Rehabilitation methods for cognitive outcomes}

Cognitive outcomes were measured in one review only. ${ }^{7}$ Psychosocial interventions and practical reminders were adequate coping strategies towards cognitive dysfunction but the meta-ethnography review also found cultural differences in coping strategies, such as Asian women being more likely to use complementary medicine like medicinal herbs, to improve cognitive functioning.

\section{Rehabilitation methods on occupational outcomes}

There were inconclusive outcomes with occupational rehabilitation $^{41}$ - whether rehabilitation consisting of counseling or exercise would indeed decrease time needed to return to work in breast cancer survivors. However, the review showed that the extensiveness of the surgical procedures correlates with the length of time needed to return to work.

\section{Discussion}

\section{An over-emphasis on physical dysfunction}

Effectiveness of rehabilitation methods on physical, psychosocial, cognitive, and occupational outcomes vary according to numerous type/s of rehabilitation methods used. Reviews investigating physical outcomes dominate the literatures. There were relatively less systematic reviews on cognitive outcomes and occupational outcomes, both were reviewed by Selamat et $\mathrm{al}^{7}$ and Hoving et $\mathrm{al}^{41}$ respectively and both suggests a lack of work and acknowledgment by health professionals and survivors in this area of cognitive impairment. Exercise was found to be effective in improving shoulder mobility, limb strength, and wound healing, although it was found to be inconclusive for fatigue and body composition (ie, BMI) management, and lymphedema. In fact with the common fatigue post operation, more work is needed as there were inconclusive findings for psycho-education, CBT, and social-emotional rehabilitation for fatigue management. There were also inconclusive results for the efficacy of complementary rehabilitation (acupuncture, yoga, art therapy, or relaxation training) on hot flashes.

Overall, exercise seems to be the one rehabilitation method to improve the narrow physical outcomes eg, for shoulder mobility, and irrespective of the type of exercise implemented. The benefit of broader exercise such as exercise for lifestyle redesign for a preventive stance 


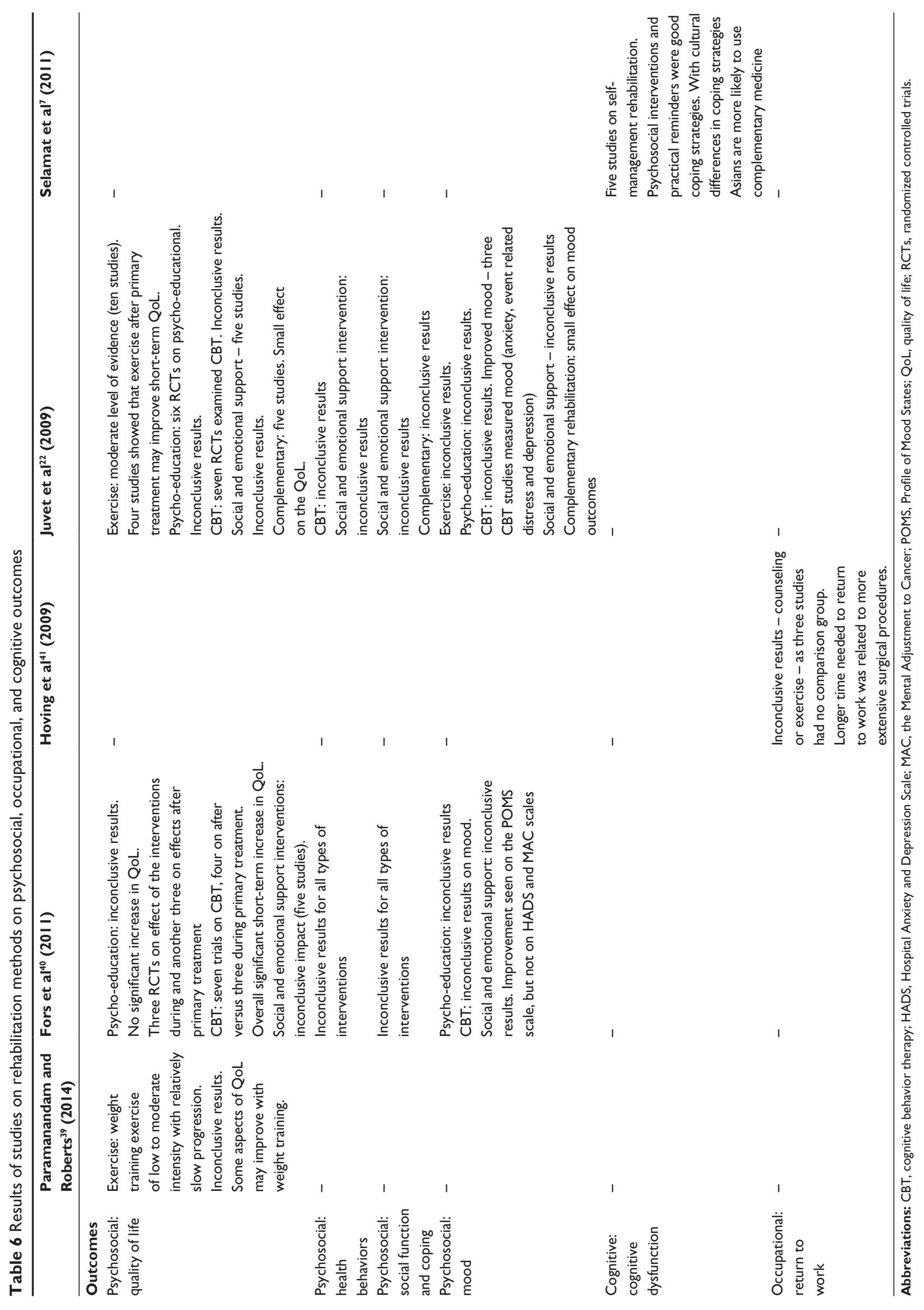


against cancer recurrence and for better QoL, needs more research. There are some studies, both quantitative and qualitative ${ }^{49,50}$ which have highlighted barriers to exercise 5 years after diagnosis of breast cancer and uncovered many expressed psychological barriers (eg, low motivation, dislike of gym), environmental barriers (eg, employment-priority, low access to facilities, interfering seasonal weather, traffic congestion to get to the gym), and lack of time. As such, studies are also needed on interventions to overcome these barriers to exercise and to ensure adherence to exercise regimes to gain its benefit on cancer recurrences and for better quality of living during the survivorship phases.

\section{A lack of evidence for non-physical rehabilitation methods}

Reviews showed inconclusive results were found for the efficacy of rehabilitation methods using psycho-education, CBT, social-emotional support, complementary-alternative methods towards improving health behaviors and/or QoL. In general, the review of the reviews also found inconclusive results on social functioning, coping, and mood outcomes. However, complementary intervention may have a small effect on mood outcomes. Nevertheless, the studies are largely heterogeneous in terms of type, length, and components of rehabilitation methods which make the comparisons rather difficult to carry out.

For less recognized problems faced by survivors of breast cancer, qualitative research identified "chemo-brain", and attitudinal changes towards work. ${ }^{7,51}$ Psycho-social rehabilitation and practical reminders were strategies proposed to improve cognitive function despite variations due to cultural differences. With occupational rehabilitation, inconclusive results were found on whether exercise or counseling rehabilitation would decrease time needed to return to work in women with breast cancer. ${ }^{41}$ Qualitative findings from focus groups of multi-ethnic survivors have highlighted several barriers such as fear of environmental hazards, high jobdemand, intrusive thoughts and family over-protectiveness, ${ }^{30}$ as well as other socio-demographic factors eg, education, range of treatment received, strenuous physical work, fatigue, and psychological factors such as negative mood. ${ }^{52}$ Future occupational studies should investigate the breadth and depth of rehabilitation methods (eg, work stamina, tolerance, psychological factors for facilitating work re-entry, work accommodations such as flexibility towards work hours etc) for enabling post-operation survivors to return to work in a design that has a control or comparison group (ie, other types of interventions, wait-list, etc).

\section{A need for more comprehensive methods to enable living for indefinite period}

Overall, the lack of evidence for non-physical rehabilitation methods highlight the lack of research work that extend beyond the rehabilitation methods for physical after-effects. The gradual acknowledgment that breast cancer is taking a form of chronic illness ${ }^{53}$ is not proportionate to the current rehabilitation methods which suggest an overall management of breast cancer as an acute/fatal condition. Amongst the important implications of this current review is that rehabilitation for women with breast cancer should be comprehensive (ie, broader rather than eg, narrowly focused on upper limb function) and proactive (rather than reactive). This stance is a better preparation of breast cancer survivors to live indefinitely with the condition and to empower them to re-engage in lifestyle modification and/ or lifestyle redesign, ${ }^{54}$ in order to address ill-health, and improve their wellbeing, lifespan, and QoL. The specific, but predominantly performance component rehabilitation, such as improving shoulder mobility, is effective but not sufficient to enable or inform survivors to live the best they can for the remainder of their life span. The lack of emphasis on patient self-management and occupational redesign towards a healthier lifestyle suggests a dire lack of focus on these broader aspects of life. This also showed a lack of appreciation that breast cancer is evolving into a form of chronic disease requiring a brand new platform to support its increasing numbers of survivors.

\section{Limitations}

The main limitation of this systematic review on systematic reviews is the difficulty to synthesize because of the heterogeneous nature of the methodology of each review. There were varying inclusion-exclusion criteria, different outcome measures, which leads to difficulties extracting and synthesizing the data.

\section{Conclusion}

In conclusion, the current rehabilitation methods tend to focus narrowly on performance components (particularly on physical impairments or dysfunctions). The review found evidence that exercise rehabilitation methods improves physical outcome post operation, although, inconclusive results exist on rehabilitation methods to improve the non-physical sequelae such as psychosocial, cognitive, occupational, and broader lifestyle performance factors. Clearly missing are the rehabilitation methods to enable survivors to redesign their lifestyle in tandem with living with a breast cancer 
condition that is taking a form of chronic disease. This calls for health prevention and illness prevention lifestyle strategies to i) control cancer recurrence and ii) to promote better QoL during the indefinite period of survivorship. With the overwhelming strong evidence that cancer risk is affected by lifestyle, future studies with higher methodological rigor should be conducted on health promotion strategies to enable healthy lifestyle.

\section{Acknowledgment}

English language checked by Dr Gail Boniface, Cardiff University, United Kingdom.

\section{Disclosure}

The authors declare no conflict of interest.

\section{References}

1. Ferlay J, Shin HR, Bray F, Forman D, Mathers C, Parkin DM. Estimates of worldwide burden of cancer in 2008: GLOBOCAN 2008. Int $J$ Cancer. 2010;127(12):2893-2917.

2. cancer.org [homepage on the Internet]. American Cancer Society Cancer Facts and Figures 2012. Atlanta: American Cancer Society. Available from: http://www.cancer.org/research/cancerfactsfigures/ cancerfactsfigures/cancer-facts-figures-2012. Accessed January 31, 2015.

3. Marín ÁP, Sánchez AR, Arranz EE, Auñón PZ, Barón MG. Adjuvant chemotherapy for breast cancer and cognitive impairment. South Med J. 2009;102(9):929-934.

4. Meade E, Dowling M. Early breast cancer: diagnosis, treatment and survivorship. Br J Nurs. 2012;21(17):S4-S8.

5. Selamat MH, Loh SY, Mackenzie L, Vardy J. Chemobrain Experienced by Breast Cancer Survivors: A Meta-Ethnography Study Investigating Research and Care Implications. PloS One. 2014;9(9):e108002.

6. Veronesi U, Cascinelli N, Mariani L, et al. Twenty-year follow-up of a randomized study comparing breast-conserving surgery with radical mastectomy for early breast cancer. $N$ Engl J Med. 2002;347(16): $1227-1232$

7. Fisher B, Anderson S, Bryant J, et al. Twenty-year follow-up of a randomized trial comparing total mastectomy, lumpectomy, and lumpectomy plus irradiation for the treatment of invasive breast cancer. $N$ Engl J Med. 2002;347(16):1233-1241.

8. Chung C, Lee S, Hwang S, Park E. Systematic Review of Exercise Effects on Health Outcomes in Women with Breast Cancer. Asian Nurs Res (Korean Soc Nurs Sci). 2013;7(3):149-159.

9. Hayes SC, Johansson K, Stout NL, et al. Upper-body morbidity after breast cancer: incidence and evidence for evaluation, prevention, and management within a prospective surveillance model of care. Cancer. 2012;118(8 Suppl):2237-2249.

10. Bosompra K, Ashikaga T, Obrien PJ, Nelson L, Skelly J. Swelling, numbness, pain, and their relationship to arm function among breast cancer survivors: a disablement process model perspective. Breast $J$. 2002;8(6):338-348.

11. Kim T, Giuliano AE, Lyman GH. Lymphatic mapping and sentinel lymph node biopsy in early-stage breast carcinoma. Cancer. 2006; 106(1):4-16.

12. Rietman JS, Dijkstra PU, Hoekstra HJ, et al. Late morbidity after treatment of breast cancer in relation to daily activities and quality of life: a systematic review. Eur J Surgical Oncol. 2003;29(3):229-238.

13. Wefel JS, Lenzi R, Theriault R, Buzdar AU, Cruickshank S, Meyers CA. 'Chemobrain' in breast carcinoma?: a prologue. Cancer. 2004;101(3): 466-475.
14. Bradley CJ, Bednarek HL, Neumark D. Breast cancer survival, work, and earnings. J Health Econ. 2002;21(5):757-779.

15. Bradley CJ, Neumark D, Bednarek HL, Schenk M. Short-term effects of breast cancer on labor market attachment: results from a longitudinal study. J Health Econs. 2005;24(1):137-160.

16. Taskila T, Martikainen R, Hietanen P, Lindbohm ML. Comparative study of work ability between cancer survivors and their referents. Eur J Cancer. 2007;43(5):914-920.

17. Morrow GR, Roscoe JA, Hickok JT, Andrews PL, Matteson S. Nausea and emesis: evidence for a biobehavioral perspective. Support Care Cancer. 2002;10(2):96-105.

18. Spencer SM, Lehman JM, Wynings C, et al. Concerns about breast cancer and relations to psychosocial well-being in a multiethnic sample of early-stage patients. Health Psychol. 1999;18(2):159-168.

19. van't Spijker A, Trijsburg RW, Duivenvoorden H. Psychological sequelae of cancer diagnosis: a meta-analytic review of 58 studies after 1980. Psychosom Med. 1997;59(3):280-293.

20. Fu MR, Chen CM, Haber J, Guth AA, Axelrod D. The effect of providing information about lymphedema on the cognitive and symptom outcomes of breast cancer survivors. Ann Surg Oncol. 2010;17(7): $1847-1853$

21. Inagaki M, Yoshikawa E, Matsuoka Y, et al. Smaller regional volumes of brain gray and white matter demonstrated in breast cancer survivors exposed to adjuvant chemotherapy. Cancer. 2007;109(1): 146-156.

22. Juvet LK, Elvsaas I, Leivseth G, et al. Rehabilitation of breast cancer patients. Norwegian Knowledge Centre for the Health Services. 2009:2.

23. Torres Lacomba M, Yuste Sánchez MJ, Zapico Goñi Á, et al. Effectiveness of early physiotherapy to prevent lympho-edema after surgery for breast cancer: randomised, single blinded, clinical trial. $B M J$. 2010;340:b5396.

24. Beurskens CH, van Uden CJ, Strobbe LJ, Oostendorp RA, Wobbes T. The efficacy of physiotherapy upon shoulder function following axillary dissection in breast cancer, a randomized controlled study. $B M C$ Cancer. 2007;7(166).

25. Schmitz KH, Ahmed RL, Troxel A, et al. Weight lifting in women with breast-cancer-related lymphedema. $N$ Engl J Med. 2009;361(7): 664-673.

26. Courneya KS, Segal RJ, Mackey JR, et al. Effects of aerobic and resistance exercise in breast cancer patients receiving adjuvant chemotherapy: a multicenter randomized controlled trial. J Clin Oncol. 2007;25(28):4396-4404.

27. Antoni MH, Lechner SC, Kazi A, et al. How stress management improves quality of life after treatment for breast cancer. $J$ Consult Clin Psychol. 2006;74(6):1143-1152.

28. Coleman AE, Tulman L, Samarel N, et al. The effect of telephone social support and education on adaptation to breast cancer during the year following diagnosis. Oncol Nurs Forum. 2005;32(4):822-829.

29. Loh SY, Yip CH, Packer T, Quek KF. Self-management pilot study on women with breast cancer: lessons learnt in Malaysia. Asian Pac J Cancer Prev. 2010;11(5):1293-1299.

30. Tan FL, Loh SY, Su T, Veloo VW, Ng LL. Return to work in multiethnic breast cancer survivors-a qualitative inquiry. Asian Pac J Cancer Prev. 2012;13(11):5791-5797.

31. Blackburn GL, Wang KA. Dietary fat reduction and breast cancer outcome: results from the Women's Intervention Nutrition Study (WINS). Am J Clin Nutr. 2007;86(3):s878-s881.

32. Zhou K, Li X, Li J, et al. A clinical randomized controlled trial of music therapy and progressive muscle relaxation training in female breast cancer patients after radical mastectomy: Results on depression, anxiety and length of hospital stay. Eur J Oncol Nurs. 2014;pii:S14623889(14)00106-9.

33. Banerjee B, Vadiraj HS, Ram A, et al. Effects of an integrated yoga program in modulating psychological stress and radiation-induced genotoxic stress in breast cancer patients undergoing radiotherapy. Integr Cancer Ther. 2007;6(3):242-250. 
34. Demark-Wahnefried W, Case LD, Blackwell K, et al. Results of a diet/ exercise feasibility trial to prevent adverse body composition change in breast cancer patients on adjuvant chemotherapy. Clin Breast Cancer. 2008;8(1):70-79.

35. Smith V, Devane D, Begley CM, Clarke M, Higgins S. A systematic review and quality assessment of systematic reviews of randomised trials of interventions for preventing and treating preterm birth. Eur J Obstet Gynecol Reprod Biol. 2009;142(1):3-11.

36. Shea BJ, Grimshaw JM, Wells GA, et al. Development of AMSTAR: a measurement tool to assess the methodological quality of systematic reviews. BMC Med Res Methodol. 2007;7:10.

37. Chan DN, Lui LY, So WK. Effectiveness of exercise programmes on shoulder mobility and lymphoedema after axillary lymph node dissection for breast cancer: systematic review. J Adv Nurs. 2010;66:1902-1914.

38. McNeely ML, Campbell K, Ospina M, et al. Exercise interventions for upper-limb dysfunction due to breast cancer treatment. Cochrane Database Syst Rev. 2010;(6):CD005211.

39. Paramanandam VS, Roberts D. Weight training is not harmful for women with breast cancer-related lymphoedema: a systematic review. J Physiother. 2014;60(3):136-143.

40. Fors EA, Bertheussen GF, Thune I, et al. Psychosocial interventions as part of breast cancer rehabilitation programs? Results from a systematic review. Psychooncology. 2011;20(9):909-918.

41. Hoving JL, Broekhuizen MLA, Frings-Dresen MH. Return to work of breast cancer survivors: a systematic review of intervention studies. BMC Cancer. 2009;9(1):117.

42. E Lima MT, E Lima JG, de Andrade MF, Bergmann A. Low-level laser therapy in secondary lymphedema after breast cancer: systematic review. Lasers Med Sci. 2014;29(3):1289-1295.

43. Huang TW, Tseng SH, Lin CC, et al. Effects of manual lymphatic drainage on breast cancer-related lymphedema: a systematic review and meta-analysis of randomized controlled trials. World J Surg Oncol. 2013;11:15.
44. Johannsen M, Farver I, Beck N, Zachariae R. The efficacy of psychosocial intervention for pain in breast cancer patients and survivors: a systematic review and meta-analysis. Breast Cancer Res Treat. 2013; 138(3):675-690.

45. Khan F, Amatya B, Ng L, Demetrios M, Zhang NY, Turner-Stokes L. Multidisciplinary rehabilitation for follow-up of women treated for breast cancer. Cochrane Database Syst Rev. 2012:12:CD009553.

46. Markes M. Exercise for Women Receiving Adjuvant Therapy of Breast-Cancer: A Systematic Review. [doctoral dissertation]. Technical University of Berlin: Berlin, Germany; 2011.

47. Moseley AL, Piller NB, Carati CJ. The effect of gentle arm exercise and deep breathing on secondary arm lymphedema. Lymphology. 2005;38(3):136-145.

48. Shamley DR, Barker K, Simonite V, Beardshaw A. Delayed versus immediate exercises following surgery for breast cancer: a systematic review. Breast Cancer Res Treat. 2005;90(3):263-271.

49. Hefferon K, Murphy H, McLeod J, Mutrie N, Campbell A. Understanding barriers to exercise implementation 5-year post-breast cancer diagnosis: a large-scale qualitative study. Health Educ Res. 2013;28(5):843-856.

50. Loh SY, Chew SL, Quek KF. Physical activity engagement after breast cancer: Advancing the health of survivors. Health. 2013;5:838-846.

51. Kennedy F, Haslam C, Munir F, Pryce J. Returning to work following cancer: a qualitative exploratory study into the experience of returning to work following cancer. Eur J Cancer Care (Engl). 2007;16(1):17-25.

52. Islam T, Dahlui M, Majid HA, et al. Factors associated with return to work of breast cancer survivors: a systematic review. BMC Public Health. 2014;14 Suppl 3:S8

53. Loh SY, Yip CH. Breast cancer as a chronic illness: implications for rehabilitation and medical education. Journal of the University of Malaya Medical Centre. 2006;9(2):3-11.

54. Lee JE, Loh SY. Physical Activity and Quality of Life of Cancer Survivors: A Lack of Focus for Lifestyle Redesign. Asian Pac J Cancer Prev. 2013;14(4):2551-2555. 


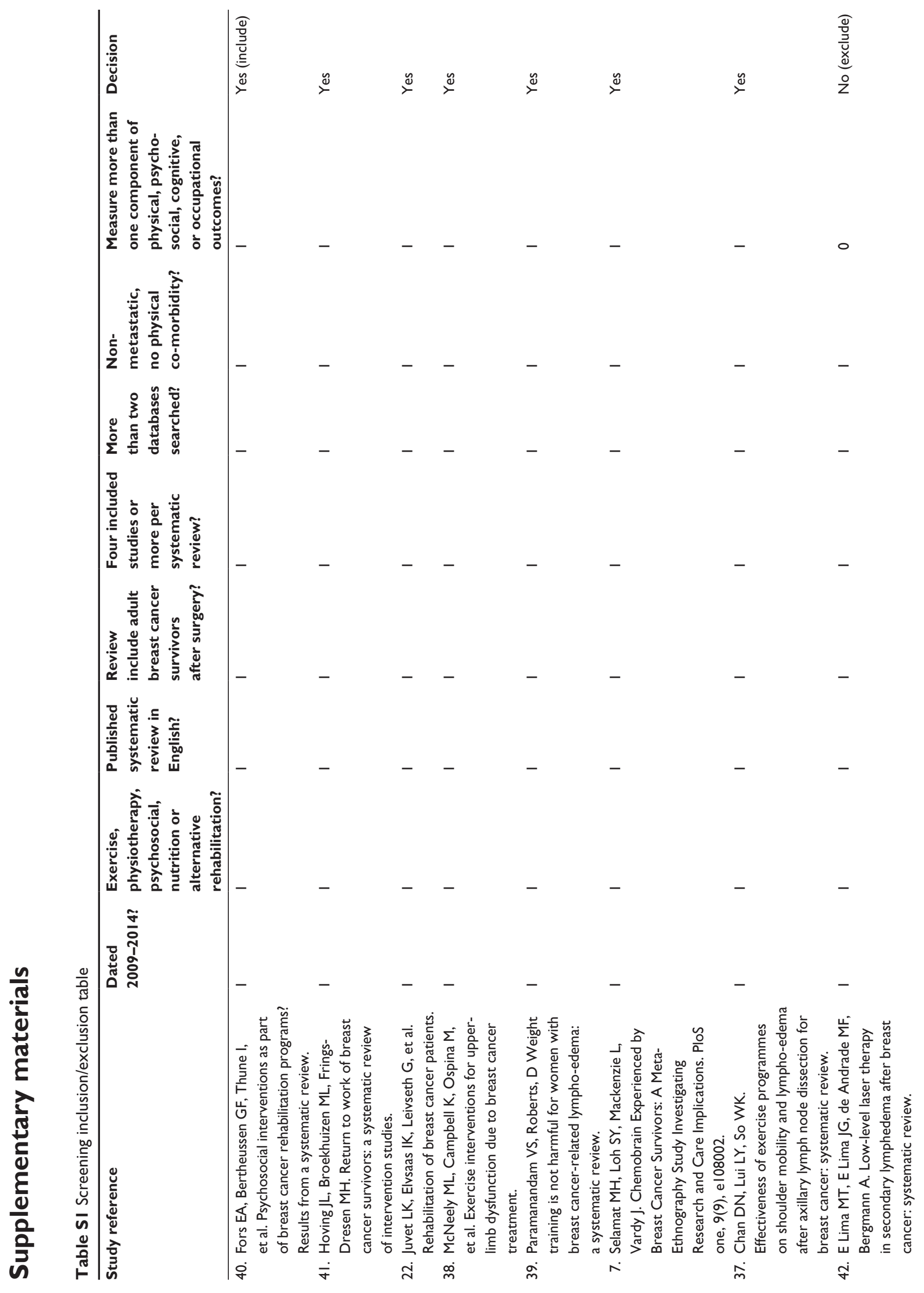




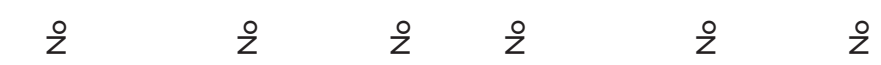

$\circ$

$\circ$

$\circ$

$-$

$-$

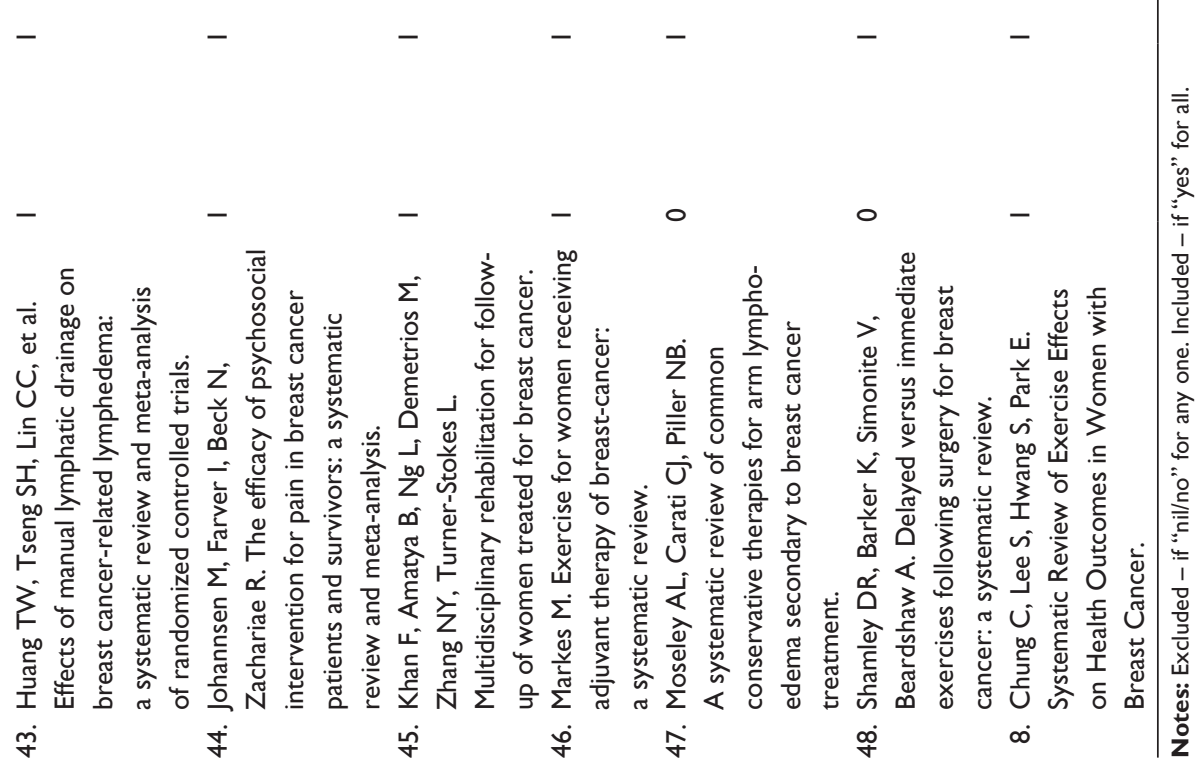

Breast Cancer: Targets and Therapy 2015:7

submit your manuscript | www.dovepress.com 
Table S2 Search strategy

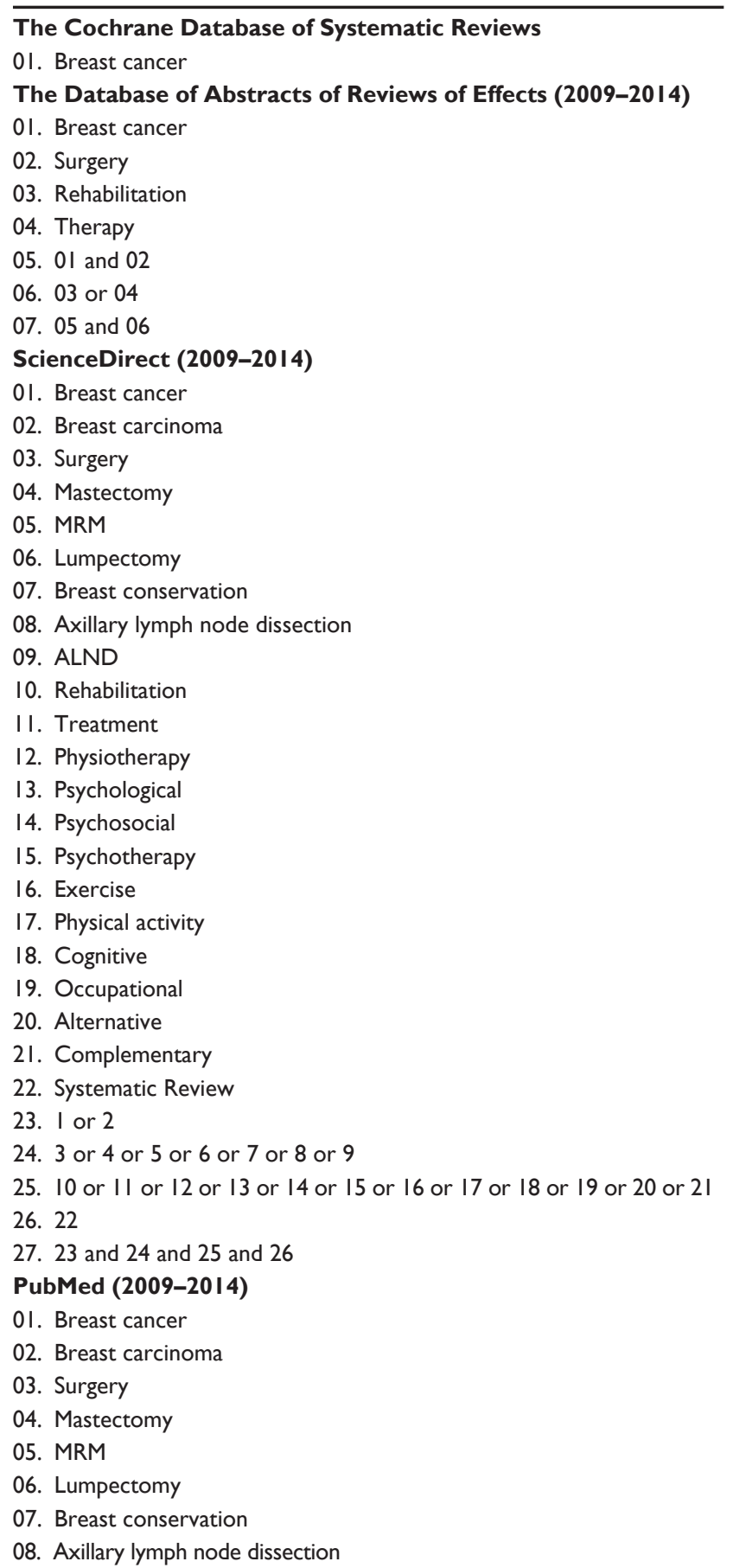

Table S2 (Continued)
09. ALND
10. Rehabilitation
II. Treatment
12. Physiotherapy
13. Psychological
14. Psychosocial
15. Psychotherapy
16. Exercise
17. Physical activity
18. Cognitive
19. Occupational
20. Alternative
21. Complementary
22. Systematic Review
23. I or 2
24. 3 or 4 or 5 or 6 or 7 or 8 or 9
25. 10 or 11 or 12 or 13 or 14 or 15 or 16 or 17 or 18 or 19 or 20 or 21
26. 22
27. 23 and 24 and 25 and 26

Abbreviations: ALND, axillary lymph node dissection; MRM, modified radical mastectomy.

\section{Publish your work in this journal}

Breast Cancer: Targets and Therapy is an international, peerreviewed open access journal focusing on breast cancer research, identification of therapeutic targets and the optimal use of preventative and integrated treatment interventions to achieve improved outcomes, enhanced survival and quality of life for the cancer patient.

\section{Dovepress}

View the full aims and scopes of this journal here. The manuscript management system is completely online and includes a very quick and fair peer-review system, which is all easy to use. Visit http:// www.dovepress.com/testimonials.php to read real quotes from published authors. 